\title{
ASSISTÊNCIA DE ENFERMAGEM FRENTE ÀS PRINCIPAIS COMPLICAÇÕES DO TRATAMENTO HEMODIÁLITICO EM PACIENTES RENAIS CRÔNICOS
}

\author{
Priscylla Oliveira Sena Sancho Graduanda do curso de enfermagem da Escola \\ Bahiana de Medicina e Saúde Pública.
}

Rafaelle Pereira Tavares

Graduanda do curso de enfermagem da Escola Bahiana de Medicina e Saúde Pública.

\author{
Cristiana da Costa Libório Lago Docente do curso de enfermagem da Escola Bahiana \\ de Medicina e Saúde Pública.
}

Endereço para correspondência: prisancho@hotmail.com

\begin{abstract}
Resumo
A insuficiência renal compromete a capacidade dos rins exercerem suas funções implicando, muitas vezes, na necessidade da hemodiálise para eliminar metabólitos e manter o equilíbrio hidroeletrolítico. O procedimento hemodialítico gera complicações potenciais e o enfermeiro deve estar apto a intervir em tais situações. Neste sentido, é importante refletir sobre o cuidado de enfermagem aos pacientes renais crônicos, particularmente no que se refere à qualidade da assistência, resolutividade do serviço/tratamento e educação em saúde. Diante disto, o objetivo deste estudo foi identificar as principais intervenções de enfermagem na assistência ao paciente renal crônico em tratamento hemodialítico. Trata-se de um estudo de revisão de literatura de natureza descritiva, com pesquisas nas bases de dados Scielo, LILACS e MEDLINE. Os resultados demonstraram que os cuidados de enfermagem envolvem a sistematização desde a entrada do paciente até a saída deste da sessão de hemodiálise. As principais complicações encontradas na literatura foram hipotensão e hipertensão arterial, câimbras musculares, síndrome do desequilíbrio da diálise, náuseas, vômitos, prurido, febre, calafrios e cefaleia. Concluiuse que a monitorização, detecção e intervenção de tais complicações é um diferencial para obtenção de uma assistência de enfermagem técnica, segura e de qualidade no tratamento dialítico.
\end{abstract}

Palavras-chave: Hemodiálise; Insuficiência renal crônica; Assistência de enfermagem; Tratamento.

\section{NURSING CARE FRONT MAJOR HEMODIALYSIS TREATMENT COMPLICATIONS OF CHRONIC RENAL PATIENTS}

\begin{abstract}
Renal insufficiency compromises the ability of the kidneys to perform their functions often implying in need of hemodialysis to eliminate metabolites and maintain hidroelectrolyte balance. The hemodialysis procedure generates potential complications and nurses must be able to intervene in such situations. In this sense, it is important to reflect on nursing care to chronic renal failure patients, particularly in relation to the quality of care, solving of service/ treatment and health education. Hence, the aim of this study was to identify the main interventions of nursing care to chronic renal failure patient under hemodialysis treatment. This is a descriptive literature review study, with researches from Scielo, LILACS and MEDLINE databases. The results showed that the nursery cares involve systematization since patient's entry to his exit from hemodialysis session. The main complications found in literature were hypotension and hypertension, muscle cramps, dialysis disequilibrium syndrome, nausea, vomiting, itching, fever, chills and headache. It was concluded that monitoring, detection and intervention of such complications is a differential for obtaining a technical, safe and quality nursing care in dialysis treatment.
\end{abstract}

Keywords: Hemodialysis; Chronic renal failure; Nursery care; Treatment. 


\title{
CUIDADOS DE ENFERMERÍA FRENTE A LAS PRINCIPALES COMPLICACIONES DEL TRATAMIENTO DE LA HEMODIÁLISIS EN PACIENTES RENALES CRÓNICOS.
}

\begin{abstract}
Resumen
La insuficiencia renal compromete la capacidad de los riñones de desempeñar éstos sus funciones, implicando a menudo la necesidad de la hemodiálisis para eliminar los metabolitos y para mantener el equilíbrio hidroelectrolítico. El procedimiento de la hemodiálisis genera complicaciones potenciales y la enfermera debe de ser capaz de intervenir en este tipo de situaciones. En este sentido, es importante reflexionar sobre los cuidados de enfermería a los pacientes con enfermedad renal, especialmente en relación a la calidad de la atención dada, a los resultados del servicio / tratamiento y a la educación para la salud. Por lo tanto, el objetivo de este estudio fue identificar las principales intervenciones de los cuidados de enfermería a los pacientes renales crónicos. Se trata de un estudio de revisión de la literatura descriptiva con base en los datos pesquisados en: SciELO, LILACS y MEDLINE. Los resultados demostraron que la sistematización de los cuidados de enfermería implica desde la entrada del paciente hasta la salida del mismo en el tratamento de la hemodiálisis. Las principales complicaciones halladas en la literatura de este tratamento fueron, la hipo y la hipertensión, los calambres musculares, el desequilibrio de la diálisis, las náuseas, los vómitos, el picazón, la fiebre, los escalofríos y el dolor de cabeza. Se concluyó así que la vigilancia, la detección y la intervención de tales complicaciones es imprescindible para la obtención de una técnica de cuidado de enfermería, segura y de calidad en el tratamento de la hemodiálisis.
\end{abstract}

Palabras clave: Hemodiálisis; Insuficiencia renal crónica; Cuidados de enfermeira.

\section{INTRODUÇÃO}

A doença renal crônica é uma lesão do órgão com perda progressiva e irreversível da função dos rins. Em sua fase mais avançada é definida como Insuficiência Renal Crônica (IRC), quando os rins não conseguem manter a normalidade do meio interno do paciente. Se diagnosticada precocemente, e com condutas terapêuticas apropriadas, serão reduzidos o sofrimento e os custos dos pacientes. ${ }^{(1)}$

As doenças mais comuns que lesam as diferentes estruturas dos rins são as glomerulonefrites, o diabetes mellitus, a hipertensão arterial, as infecções urinárias repetidas - que ocorrem quando há dificuldades de escoamento da urina - e presença de cálculos ou cistos renais. Algumas doenças levam anos para que seus danos se tornem aparentes. Quanto mais essas doenças progridem ou se agravam, maiores danos levam aos rins, perturbando suas funções, determinando então, a insuficiência renal. ${ }^{(2)}$

É importante considerar que a IRC tem caráter irreversível e necessita de tratamento dialítico imediato, por tempo indeterminado, com o objetivo de controlar a uremia e evitar a morte do paciente. ${ }^{(2)}$ A hemodiálise é o método de diálise mais comumente empregado para remover substâncias nitrogenadas tóxicas do sangue e excesso de água. Requer cuidado intensivo devido à possibilidade de intercorrências clínicas. Neste sentido, é importante refletir sobre o cuidado de enfermagem aos pacientes renais crônicos, particularmente no que 
se refere à qualidade da assistência, resolutividade do serviço/tratamento e educação em saúde. $^{(3)}$

A hemodiálise é o método mais empregado no Brasil para o tratamento de IRC. É a modalidade de diálise que se processa num circuito extracorpóreo formado por uma linha (set) arterial e outra venosa de material plástico, entre as quais se interpõe um rim artificial ou hemodialisador. ${ }^{(2,4)}$

Atualmente, devido ao grande processo e avanço tecnológico, as máquinas de hemodiálise apresentam maior segurança e eficácia, tornando o tratamento mais seguro. Embora haja alarmes que indiquem qualquer alteração que ocorra no sistema (detectores de bolhas, alteração de temperatura e do fluxo do sangue, entre outros), ainda não se pode garantir que as complicações deixem de ocorrer. A hemodiálise busca a reversão não somente dos sintomas urêmicos, mas também a redução das complicações que são inerentes ao próprio procedimento e a diminuição do risco de mortalidade. Por este motivo, os profissionais de enfermagem devem estar sempre atualizados para promover um tratamento com segurança e qualidade ao paciente renal crônico. ${ }^{(5)}$

A Insuficiência Renal Crônica e o tratamento hemodialítico provocam uma sucessão de situações para o paciente renal crônico que compromete não só o aspecto físico, como também o psicológico, com repercussões pessoais, familiares e sociais. A convivência com estes pacientes torna clara a importância da intervenção da enfermagem em busca de solução nas limitações provocadas pela IRC e o tratamento, sendo necessário um reaprender a viver, de uma maneira mais humana. ${ }^{(6)}$

O paciente com IRC, em programa de hemodiálise, é conduzido a conviver diariamente com uma doença incurável que o obriga a uma forma de tratamento dolorosa, de longa duração e que provoca, juntamente com a evolução da doença e suas complicações, ainda maiores limitações e alterações de grande impacto, que repercutem tanto na sua própria qualidade de vida quanto na do grupo familiar. ${ }^{(1)}$

A hemodiálise, na maioria das vezes, representa uma esperança de vida, já que a doença é um processo irreversível. Contudo, observa-se que geralmente as dificuldades de adesão ao tratamento estão relacionadas à não aceitação da doença, à percepção de si próprio e ao relacionamento interpessoal com familiares e ao convívio social. ${ }^{(7)}$

O procedimento hemodialítico gera complicações potenciais, por isso o enfermeiro deve estar apto a intervir em tais complicações, entre elas: hipotensão e hipertensão arterial, 
câimbras musculares, síndrome do desequilíbrio da diálise, náuseas, vômitos, prurido, cefaléia, febres e calafrios. ${ }^{(8)}$

O objetivo da assistência de enfermagem neste setor é identificar e monitorar os efeitos adversos da hemodiálise e complicações decorrentes da própria doença, desenvolvendo ações educativas de promoção, prevenção e tratamento. ${ }^{(9)}$ Segundo a RDC n ${ }^{\circ}$ $154^{(10)}$, de 15 de Junho de 2004, o programa de hemodiálise deve integrar em cada turno, no mínimo um enfermeiro para cada trinta e cinco pacientes.Nesse setor existem várias intervenções específicas realizadas pelo enfermeiro, entretanto,os registros despendem muito tempo, uma vez que são realizados de forma descritiva, e por isso acabam não sendo feitos em virtude da alta demanda de atendimento. ${ }^{(9)}$

As complicações que ocorrem durante a sessão de hemodiálise podem ser eventuais, mas algumas são extremamente graves e fatais. A equipe de enfermagem tem importância muito grande na observação contínua dos pacientes durante a sessão, podendo ajudar a salvar muitas vidas e evitar muitas complicações ao fazer o diagnóstico precoce de tais intercorrências. O paciente deve ter extrema confiança nos profissionais que devem sempre mostrar-se prestativos, atenciosos e sempre alertas para intervir quando necessário. ${ }^{(5)}$

O enfermeiro deve coordenar a assistência prestada, identificando as necessidades individuais de cada paciente, proporcionando meios de atendimento que visem uma melhor adequação do tratamento, garantindo assim uma qualidade de vida melhor, aproveitando todos os momentos para criar condições de mudanças quando necessário. A prática do cuidar personalizado está diretamente ligada à qualidade da assistência prestada, e uma das formas de alcançar este objetivo é através do processo de enfermagem. ${ }^{(7)}$

O estudo subsidiará o desenvolvimento de uma assistência de enfermagem adequada ao paciente hemodialítico, auxiliando na ampliação do conhecimento sobre as intervenções frente às complicações, aos cuidados hospitalares e domiciliares, ofertando aos pacientes melhores condições durante o tratamento.

O objetivo deste estudo foi identificar as principais intervenções de enfermagem na assistência ao paciente renal crônico em tratamento hemodialítico. 


\section{METODOLOGIA}

Trata-se de um estudo de pesquisa bibliográfica, baseado em produções científicas da língua portuguesa sobre o tema no período compreendido entre 1998 e 2013. Os trabalhos foram lidos em sua íntegra.

Os trabalhos de revisão são estudos que analisam a produção bibliográfica em determinada área temática, dentro de um recorte de tempo, fornecendo uma visão geral ou um relatório do estado-da-arte sobre um tópico específico, evidenciando novas ideias, métodos, subtemas que têm recebido maior ou menor ênfase na literatura selecionada. ${ }^{(11)}$

Após a definição do tema e objetivo deste trabalho, foram determinadas, a partir de pesquisa no DECs, as seguintes palavras-chave: assistência de enfermagem, insuficiência renal crônica, hemodiálise e tratamento. As buscas foram realizadas nas bases de dados Scielo, LILACS e MEDLINE, encontrando um total de 58 publicações.

Como critérios de inclusão foram utilizados: período de publicação compreendido entre 1998 e 2013, língua portuguesa, relevância do trabalho quanto ao tema proposto, resultando em um total de 16 publicações selecionadas. Após a leitura, os artigos selecionados foram organizados em forma de fichamentos para leitura e análise do conteúdo.

Além da utilização dos trabalhos acima referidos, foram também coletados livros na área de fisiologia renal, nefrologia e assistência de enfermagem aos pacientes renais crônicos, a fim de nortear este estudo.

A partir da análise dos artigos selecionados neste estudo optou-se pela apresentação dos resultados em duas categorias acerca da assistência de enfermagem na terapia hemodialítica: uma visão geral da assistência de enfermagem na avaliação pré, durante e póshemodiálise e principais complicações e assistência de enfermagem no processo de hemodiálise. A discussão foi realizada de forma descritiva para análise das categorias em questão com embasamento nos autores utilizados no estudo. 


\section{RESULTADOS E DISCUSSÃO}

\section{UMA VISÃO GERAL DA ASSISTÊNCIA DE ENFERMAGEM NA AVALIAÇÃO PRÉ, DURANTE E PÓS-HEMODIÁLISE}

Como o enfermeiro é o profissional que assiste o paciente nas sessões de hemodiálise de forma mais presente, ele deve estar apto/treinado para prontamente intervir e assim evitar outras potenciais complicações. Os cuidados de enfermagem envolvem a sistematização desde a entrada do paciente até a saída deste da sessão de hemodiálise. ${ }^{(12)}$

Quando administrada por pessoal competente e com os recursos técnicos indispensáveis, a hemodiálise é um processo terapêutico praticamente isento de riscos para a vida do paciente. Todavia, algumas complicações podem ocorrer, mesmo quando é realizada dentro da melhor técnica. ${ }^{(13)} \mathrm{O}$ profissional de enfermagem deve orientar quanto ao funcionamento da hemodiálise, terapia nutricional, ingestão de líquidos, complicações da hemodiálise e formas de prevenção, cuidados com acesso venoso, anticoagulação e seus cuidados, importância da atividade física, do lazer e da associação a grupos de apoio. ${ }^{(14)}$

Deve-se recepcionar o paciente ao chegar à unidade de diálise, sempre observando seu aspecto geral e realizando uma avaliação pré-hemodiálise, que envolve: encaminhar o paciente à balança para o registro o peso e, em seguida, encaminhar o paciente à máquina, verificar sinais vitais, orientar auxiliares e/ou técnicos a comunicar qualquer alteração para o enfermeiro responsável, conversar com o paciente sobre qualquer sintoma que ele tenha sentido desde a última diálise e se não houver restrição iniciar a sessão de diálise. ${ }^{(12)}$

O profissional de enfermagem de diálise está em assistência constante durante a hemodiálise aguda. A pressão arterial e o pulso são registrados pelo menos a cada meia hora quando a condição do paciente está estável. Todas as pressões do aparelho e velocidades do fluxo são verificadas e registradas com regularidade. O enfermeiro avalia as respostas do paciente à remoção de líquido e soluto e a condição e função do acesso vascular do paciente. $^{(15)}$

$\mathrm{Na}$ avaliação pós-hemodiálise deve-se cuidar para sinais de sangramento no local da punção venosa, checar sinais vitais, verificar o peso, não permitir que o paciente sintomático deixe a unidade sem atendimento médico, etc. ${ }^{(12)} \mathrm{A}$ evolução de enfermagem é uma avaliação global do plano de cuidados e devem ser incluídos dados relevantes, identificados durante avaliação de enfermagem, isto é: melhora, piora ou manutenção do quadro anterior, adesão às 
recomendações previamente prescritas e o aparecimento de novos problemas. Através da evolução de enfermagem pode-se avaliar a assistência prestada, bem como alterar o plano de assistência, visando ao alcance dos resultados esperados. ${ }^{(9)}$

O sangue coletado imediatamente após a diálise pode mostrar níveis falsamente baixos de eletrólitos, ureia e creatinina. Acredita-se que o processo de equilíbrio continue por algum tempo depois da diálise porque essas substâncias se movem de dentro da célula para o plasma. Para assegurar a acurácia dos resultados dos dados laboratoriais após a diálise, deve-se aguardar no mínimo duas ou três horas antes de coletar o material do paciente para exames laboratoriais. $^{(15)}$

Além da observação contínua do paciente, a verificação do sistema (capilar/linha) e os parâmetros do equipamento (pressão venosa, pressão arterial, pressão transmembrana, temperatura do dialisato, concentração de sódio e ultrafiltração) devem ser realizados pela equipe de enfermagem antes de iniciar o sistema dialítico, a fim de que a prescrição de diálise seja cumprida de forma efetiva e problemas como coagulação do sistema e baixo fluxo do acesso vascular possam ser identificados e corrigidos prontamente. Porém, esses parâmetros são verificados, novamente pelo enfermeiro no momento da visita de enfermagem. ${ }^{(9,16)}$

\section{PRINCIPAIS COMPLICAÇÕES E ASSISTÊNCIA DE ENFERMAGEM NO PROCESSO DE HEMODIÁLISE}

\section{Hipotensão arterial}

A hipotensão arterial é, sem dúvida, a principal complicação do tratamento hemodialítico, ocorrendo em até $20 \%$ das sessões. ${ }^{(17)}$ Os sinais e sintomas mais comuns são tonturas e sensação de desfalecimento, dor precordial, sudorese, confusão mental e taquicardia. ${ }^{(12)}$

As principais causas para o aparecimento da hipotensão arterial durante a sessão são: ganho excessivo de peso, hiponatremia, ultrafiltração excessiva e uso de anti-hipertensivos durante a diálise. Sedativos e tranquilizantes também podem provocar hipotensão e devem ser evitados, quando possível. ${ }^{(5,12)}$

Muitas vezes, a hipotensão ocorre sem que se registre redução aguda no volume de sangue circulante ou na taxa de reenchimento vascular. Essa observação sugere que a 
hipotensão pode ser causada pelo repentino desequilíbrio nos mecanismos de controle da pressão arterial que estariam compensando a redução do volume intravascular. ${ }^{(17)}$

Atualmente, os aparelhos de diálise ajudam na prevenção da hipotensão porque a quantidade de ultrafiltração é controlada apenas apertando-se um botão. Também é possível variar a concentração de sódio do dialisato. Um nível de sódio mais elevado no dialisato significa que menos sódio é removido do sangue. Um sódio sérico mais alto ajuda o corpo, já que ele desloca o líquido do interstício para o compartimento intravascular. ${ }^{(15)}$

Independente do mecanismo fisiopatológico envolvido o tratamento agudo, na hipotensão arterial durante a hemodiálise, implica na infusão de solução salina e agentes hipertônicos, como cloreto de sódio a $20 \%$ ou $30 \%$, manitol, glicose a $50 \%$ e albumina. Se necessário, o paciente deverá ser colocado na posição de tredelenburg. Além disso, a redução da velocidade de ultrafiltração por meio do aumento do tempo de diálise, a reavaliação do peso seco do paciente e a reorientação do paciente para reduzir o ganho de peso interdialítico podem reduzir a incidência de hipotensão arterial durante a diálise. ${ }^{(5,17)}$

Intervenções de enfermagem como o monitoramento cuidadoso dos sinais vitais e observação de sintomas específicos podem ajudar a limitar a ocorrência e a intensividade de episódios hipotensivos nesses pacientes. ${ }^{(18)}$

\section{Hipertensão arterial}

Crise hipertensiva é uma complicação pouco frequente durante a hemodiálise e sua fisiopatologia obscura. ${ }^{(17)} \mathrm{A}$ hipertensão durante a diálise geralmente é causada por excesso de sódio e água. Isso pode ser confirmado comparando-se o peso atual do paciente com o seu peso seco ou ideal. ${ }^{(15)}$

Em alguns pacientes, observa-se elevação das catecolaminas e, em outros, ativação do sistema renina-angiotensina-aldosteronasecundária a depressão de volume. A orientação para suspender a medicação anti-hipertensiva no período pré-dialítico também contribui para a elevação da pressão arterial durante a diálise. De qualquer forma, tanto a administração de nifedipina quanto de captopril são úteis no controle da crise hipertensiva induzida pela diálise. ${ }^{(17)}$ 


\section{Cãimbras musculares}

Cãimbramuscular é uma complicação frequente da hemodiálise; predominanos membros inferiores e ocorre, preferencialmente, na segunda metade da hemodiálise. Frequentemente é precedida de hipotensão arterial. ${ }^{(17)}$ Os três fatores predisponentes mais importantes na sua etiologia são: a hipotensão arterial, o paciente abaixo do peso seco e o uso de solução dialítica pobre em sódio. ${ }^{(19)}$

As cãimbras musculares podem ocorrer durante a diálise em consequência da remoção do excesso de líquido, o que resulta em volume intravascular diminuído e perfusão muscular reduzida. ${ }^{(15)}$ Nesse sentido, é necessário orientar o paciente a reduzir o ganho de peso no intervalo interdialítico e, se necessário, prolongar o tempo de diálise, a fim de tornar a ultrafiltração menos intensa. ${ }^{(17)}$

Como geralmente as cãimbras ocorrem concomitantemente com a hipotensão, podem ser tratadas diminuindo a velocidade de ultrafiltração e administrando solução de glicose ou soro fisiológico hipertônico, numa tentativa de aumentar a perfusão para os músculos. A prevenção dos episódios hipotensivos eliminaria a maior parte dos episódios de câimbras. ${ }^{(5)} \mathrm{A}$ elevação do nível de sódio do banho da diálise também pode ajudar a evitar os episódios de câimbras musculares durante e após o tratamento. ${ }^{(15)}$

\section{Síndrome do desequilíbrio da diálise}

É um conjunto de sintomas sistêmicos e neurológicos que pode vir a ocorrer durante o tratamento de diálise. Caracteriza-se por confusão mental, cefaleia, vômitos, tremores, náuseas, agitação, delírio, contrações musculares ou crises convulsivas generalizadas. ${ }^{(12,15)}$

A síndrome de desequilíbrio da diálise é causada por uma demora na transferência de ureia do cérebro para o sangue, causando um gradiente de concentração. Esse gradiente favorece a translocação de água para o interior das células do sistema nervoso central, levando ao edema cerebral. ${ }^{(15,19)}$ Ocorre em pacientes muito urêmicos na fase inicial do tratamento.Essa síndrome é frequentemente observada em pacientes que iniciam o tratamento dialítico quando crianças e naqueles submetidos a diálise de alto fluxo e alta eficiência (diálise curta). Entretanto, pode ocorrer com intensidade variável em qualquer paciente. ${ }^{(17)}$

A utilização de diálises de curta duração e baixa intensidade com redução do intervalo interdialítico está indicada nos pacientes que apresentam concentração sérica de ureia muito 
elevada ao iniciarem o tratamento dialítico. O uso de soluções hipertônicas como manitol, intermitente ou contínuo e a elevação das concentrações de sódio do dialisado são medidas que, por promoverem a elevação da osmolaridade plasmática, reduzem o edema cerebral. ${ }^{(17)}$

O tratamento para essa síndrome consiste, sobretudo, na administração profilática de medicamento anticonvulsivante no início da diálise a todo paciente com níveis excessivamente altos de ureia no sangue. Quando ocorrem convulsões, o paciente deve ser sedado e a diálise suspensa, podendo ser reiniciada algumas horas depois, se sua condição clínica permitir. A sua duração, em geral, é cerca de $12 \mathrm{~h}$, sendo rara a persistência de sintomas por tempo mais prolongado. O seu prognóstico geralmente é bom. ${ }^{(17,20)}$

\section{Náuseas e vômitos}

Náuseas e vômitos são ocorrências comuns e ocorrem em até 10\% dos tratamentos rotineiros de diálise, sendo sua etiologia multifatorial. Grande parte desses episódios está relacionada à hipotensão arterial. ${ }^{(21)}$ A ocorrência de náuseas e vômitos pode estar associada a outras complicações clínicas, tais como síndrome do desequilíbrio da diálise, cefaleia e reação a produtos utilizados na hemodiálise. ${ }^{(16)}$

Cabe à equipe de enfermagem comunicar ao médico e administrar as medicações prescritas. Deve-se tratar primeiramente a hipotensão, quando presente. Caso as náuseas e vômitos persistam, pode-se administrar um antiemético. ${ }^{(21)}$

É importante lembrar que a ocorrência de vômitos durante a hemodiálise pode levar a broncoaspiração, dependendo da posição e do grau de consciência do paciente no momento do evento, devendo então o mesmo ser posicionado em decúbito lateral oposto ao acesso vascular. $^{(16)}$

A remoção de próteses dentárias pode se fazer necessária. Medidas de higiene e conforto, como limpeza das vestimentas e enxágue da boca, devem ser realizadas assim que o cliente apresentar melhora do quadro. Deve-se estar atento para os valores da PA, lembrando que o vômito leva a um estímulo do nervo vago, o que resulta em um valor aumentado da PA; portanto, deve-se aguardar de 10 a 15 minutos para uma nova aferição, certificando-se dos valores obtidos. ${ }^{(16)}$ 


\section{Prurido}

Embora aceito como uma das complicações da insuficiência renal crônica, do tratamento dialítico de longa duração, da presença de hiperparatieroidismo secundário e de alterações do metabolismo do cálcio e do fósforo, o prurido pode, em alguns pacientes, iniciar-se ou agravar-se durante a sessão de hemodiálise. ${ }^{(17)}$

Atualmente, essa é uma complicação pouco frequente durante a hemodiálise, graças à melhora dos métodos de esterilização dos dialisadores e linhas e da qualidade dos anticoagulantes utilizados na hemodiálise. ${ }^{(16)}$ Tem sido atribuído ao efeito tóxico da uremia na pele. As toxinas urêmicas circulantes são responsáveis pelo prurido, que pode desaparecer como o inicio do tratamento de hemodiálise. Entretanto, vale ressaltar que nem sempre a hemodiálise alivia, podendo, inclusive, piorá-lo. ${ }^{(21)}$

Alguns tratamentos são eficazes como emolientes tópicos à base de cânfora, aplicação de ultravioleta, uso de carbonato de cálcio (quando o produto cálcio-fósforo for elevado), dieta para controle do fósforo, uso de anti-histamínicos por via oral ou endovenosa e a paratireoidectomia (para os pacientes com osteodistrofia e hiperparatireoidismo grave). ${ }^{(19,21)}$

O uso de anti-histamínicos durante o tratamento poderá ser indicado pelo médico. Cabe à equipe de enfermagem orientar sobre os cuidados com a pele, como hidratação e banhos com sabonete à base de glicerina para melhora dos sintomas. Os pacientes devem ser aconselhados a tomar banhos rápidos e com água em temperatura ambiente, além de utilizarem cremes hidratantes. ${ }^{(17)}$

\section{Febre e calafrios}

O paciente renal crônico é imunodeprimido e, consequentemente, tem uma suscetibilidade aumentadaà infecções. ${ }^{(19)}$ Os agentes causadores de bacteremias em pacientes submetidos à hemodiálise podem estar associados à contaminação do dialisato ou do sistema hidráulico, às técnicas inadequadas de desinfecção dos equipamentos de diálise ou ao reprocessamento do dialisador. As bacteremias podem causar endocardite, meningite e osteomielite. $^{(16)}$

Febre de baixa intensidade durante a hemodiálise pode estar relacionada a pirogênios presentes na solução dialítica e não a uma infecção verdadeira. O tempo de evolução da febre pode ser útil para a distinção entre reação pirogênica e infecção. Os pacientes com febre 
relacionada ao pirogênio são afebris antes da diálise, mas tornam-se febris durante a diálise; além disso, a febre desaparece espontaneamente após o término da diálise. Os pacientes com septicemia relacionada ao local de acesso frequentemente são febris antes da instituição da diálise e, na ausência do tratamento, a febre persiste durante e após a diálise. ${ }^{(5)}$

Os procedimentos adotados nos pacientes que apresentam febre no período dialítico são: verificação da temperatura do paciente e o apontado pela máquina de hemodiálise e realizar coleta de amostra para hemocultura. ${ }^{(19)}$ A equipe de enfermagem deve estar atenta às queixas do paciente durante a diálise, pois muitas vezes o mesmo apresenta tremores e calafrios sem, no entanto, se identificar elevação da temperatura axilar. Mesmo assim, o médico deverá ser avisado. ${ }^{(16)}$

O tratamento é realizado com administração de antitérmicos e antibióticos. Vale ressaltar que algumas clínicas tem como rotina a coleta de amostra da água da hemodiálise para cultura. ${ }^{(5)}$ No caso do tratamento de uma presumível infecção do acesso vascular em um paciente de diálise febril com cateter temporário (subclávio, jugular interno ou femoral), caso não exista fonte óbvia de infecção, devem ser realizadas culturas sanguíneas e também a remoção do cateter. $\mathrm{O}$ atraso na remoção de um cateter infectado pode resultar em complicações sépticas que poderiam ser evitadas. ${ }^{(19)}$

\section{Cefaleia}

Diversas variáveis estão envolvidas no procedimento dialítico e a gênese da cefaleia provavelmente é multifatorial em muitos casos. Há dificuldades em sua classificação, caracterização clínica, entendimento dos mecanismos fisiopatológicos e poucas evidências suportando as condutas atualmente realizadas. ${ }^{(22)}$

As causas mais encontradas são: hipertensão e hipotensão arterial, alterações no peso corporal e ansiedade. Essa complicação pode estar associada a aumento das concentrações de bradicinina e óxido nítrico durante a hemodiálise, síndrome do desequilíbrio ou manifestação da abstinência de cafeína em pacientes que ingerem muitocafé, uma vez que a diálise retira essa substância. No caso de pacientes com cefaleia de repetição, a investigação de hemorragia subdural ou intracraniana, que são complicações pouco frequentes, deve ser realizada. ${ }^{(5,16,20)}$

A equipe de enfermagem deverá verificar a PA, comunicar a queixa ao médico, juntamente com o valor da PA, e medicar conforme prescrição (analgésicos por via oral ou endovenosa são comumente prescritos). Além disso, devemos proporcionar ao paciente um 
ambiente o mais tranquilo possível, evitando luz direta e barulho excessivo. Assim como nos casos de náuseas e vômitos, uma redução na velocidade de fluxo sanguíneo durante a parte inicial da diálise pode ser tentada. ${ }^{(5,16)}$

\section{CONSIDERAÇÕES FINAIS}

A compreensão das complicações da hemodiálise e do funcionamento do seu circuito é indispensável para que o enfermeiro realize uma assistência segura e de qualidade, compreendendo o processo de monitorização, detecção e rápida intervenção.

Após a realização deste estudo, pode-se perceber que as principais complicações apresentadas pelo paciente em tratamento hemodialítico são hipotensão e hipertensão arterial, câimbras musculares, síndrome do desequilíbrio da diálise, náuseas, vômitos, prurido, febre, calafrios e cefaleia, e que o enfermeiro é o profissional que está mais próximo dos pacientes renais crônicos nas sessões de hemodiálise mantendo uma estreita relação com eles. Por esse motivo, este profissional deve está apto para intervir e evitar qualquer intercorrência que pode acontecer mesmo com todos os recursos tecnológicos avançados ou com a adequação estrutural dos serviços de hemodiálise.

A sistematização e conduta de enfermagem no tratamento hemodialítico consistem na participação da realização das terapias dialíticas quando essas forem indicadas, na priorização de ações durante os episódios de complicações e na observação do progresso do paciente e resposta ao tratamento. A conscientização dos pacientes sobre a importância da mudança no estilo de vida e comportamento, dando ênfase em suas restrições e atribuições no tratamento, é uma estratégia de educação em saúde do enfermeiro para a prevenção de intercorrências.

Diante do exposto neste trabalho, nota-se a necessidade da realização de novos estudos sobre o tema, uma vez quese verificou uma carência muito grande de trabalhos escritos, principalmente por profissionais de enfermagem, sobre as complicações do tratamento hemodialítico no renal crônico, buscando as intervenções de enfermagem tanto de forma curativas como preventivas. Desse modo, conclui-se que todas as ações adequadas desenvolvidas pelo enfermeiro às diferentes situações na avaliação clínica do paciente em tratamento dialítico terão como resultado final uma assistência de enfermagem técnica, de qualidade e segura. A monitorização, detecção e intervenção de tais complicações é um diferencial para a realização de um procedimento dialítico com segurança. 


\section{REFERÊNCIAS}

1. Higa K, Kost MT, Morais MC, Polins BRG, Soares DM. Qualidade de vida de pacientes portadores de insuficiência renal crônica em tratamento de hemodiálise. Acta Paul Enferm. 2008;21(Número Especial):203-6.

2. Chagas NR, Freitas MCde, Leite ACdeS, Monteiro ARM, Ramos IC. A Teoria De Orem E O Cuidado A Paciente Renal Crônico. R Enferm UERJ, Rio de Janeiro, 2007 Abr/Jun; 15(2):444-9.

3. Rodrigues TA, Botti NCL. Cuidar e o ser cuidado na hemodiálise. Acta Paul Enferm. 2009;22(Especial-Nefrologia):528-30.

4. Mota A. Significado da liberdade e da responsabilidade pelo cuidar de si e do cliente em hemodiálise [dissertação]. [Salvador]: Universidade Federal da Bahia; 2005. 159 p.

5. Marques IR, Nascimento CD. Intervenções de enfermagem nas complicações mais frequentes durante a sessão de hemodiálise: revisão da literatura. Rev Bras Enferm. 2005 Nov/Dez; 58(6):719-22.

6. Casagrande LDR, Cesarino CB. Paciente com Insuficiência Renal Crônica em Tratamento Hemodialítico: Atividade Educativa do Enfermeiro. Rev. Latino-Am. Enferm. 1998 Out;6(4):31-40.

7. Lopes MHBdeM, Martino MMFde, Souza EFde . Diagnósticos de enfermagem em pacientes com tratamento hemodialítico utilizando o modelo teórico de Imogene King. Rev Esc Enferm USP 2007;41(4):629-35.

8. Silva GLDFda, Thomé EGdaR. Complicações no Procedimento Hemodialítico em Pacientes com Insuficiência Renal Aguda: Intervenções de Enfermagem. Rev. Gaúcha Enferm. 2009 Mar;30(1):33-9.

9. Lima LCEQde, Oliveira SMde, Pinto MH, Poletti NAA, Ribeiro DF, Ribeiro RdeCHMR. Elaboração de um instrumento da assistência de enfermagem na unidade de hemodiálise. Acta Paul Enferm. 2008;21(Número Especial):169-73.

10. Melo DRde. Resolução da diretoria colegiada-RDC nº. 154, de 15 de junho de 2004. [Acesso em 2013 nov 19]. Disponível em:

http://www.anvisa.gov.br/hotsite/segurancadopaciente/documentos/rdcs/RDC $\% 20 \mathrm{~N} \% \mathrm{C} 2 \% \mathrm{~B}$ A\%20154-2004.pdf

11. Noronha DP, Ferreira SMSP. Revisões de literatura. In: Campello BS, Condón BV, Kremer JM, organizadores. Fontes de informação para pesquisadores e profissionais. Belo Horizonte: UFMG; 2000. p.191.

12. Araújo ACS, Santo EE. A importância das intervenções do enfermeiro nas intercorrências durante a sessão de hemodiálise. Caderno Saúde e Desenvolvimento. 2012 Jul/Dez;1(1):4458.

Revista Enfermagem Contemporânea. 2013 Dez;2(1):169-183 
13. Furtado AM, Pennafort VPS, Silva LF, Silveira LC, Freitas MC, Queiroz MVO. Cuidar permanência: enfermagem 24 horas, nossa maneira de cuidar. Rev Bras Enferm. 2010 Nov/Dez;63(6):1071-6.

14. Santos Idos, Rocha RPF, Berardinelli LMM. Necessidade de orientação de enfermagem para o autocuidado de clientes em terapia de hemodiálise. Rev Bras Enferm. 2011 MarAbr;64(2):335-42.

15. Morton PG, Fontaine DK. Cuidados críticos de enfermagem: uma abordagem holística. 9a . ed. Rio de Janeiro: Guanabara Koogan; 2011. p. 748- 49.

16. Cruz SCGR, Oliveira SdeC, Matsui T. Terapia renal substitutiva. $1^{a}$. ed. São Paulo: Fundap; 2012.

17. Castro MCM. Atualização em diálise: Complicações agudas em hemodiálise. J Bras Nefrol. 2001;23(2):108-13.

18. Barros E, Manfro RC, Thomé FS, Gonçalves LFS. Nefrologia, rotinas, diagnóstico e tratamento. $2^{\text {a }}$ ed. Porto Alegre: Artmed; 1999.

19. Dalgirdas JT. Manual de diálise. $3^{\text {a }}$ ed. Rio de Janeiro: Medsi; 2003.

20. Terra FdeS, Costa AMDD, Figueiredo ET, Morais AM, Costa MD, Costa RD. As principais complicações apresentadas pelos pacientes renais crônicos durante a sessão de hemodiálise. Rev Bras Clin Med. 2010;8(3):187-92.

21. Fermi MRV. Manual de Diálise para Enfermagem. $2^{a}$ ed. São Paulo: Guanabara Koogan; 2010.

22. Antoniazzi AL, Bigal ME, Bordini CA, Speciali JG. Cefaléia relacionada à hemodiálise: análise dos possíveis fatores desencadeantes e do tratamento empregado.Arq. Neuropsiquiatr.2002;60(3-A):614-18. 